\title{
Elevated serum IgE, oral corticosteroid dependence and IL-17/22 expression in highly neutrophilic asthma
}

\author{
Michela Bullone ${ }^{1}$, Vitina Carriero ${ }^{1}$, Francesca Bertolini ${ }^{1}$, Anna Folino ${ }^{1}$, \\ Alessandro Mannelli $\mathbb{1 0}^{2}$, Antonino Di Stefano ${ }^{3}$, Isabella Gnemmi ${ }^{3}$, \\ Roberto Torchio ${ }^{4}$ and Fabio L.M. Ricciardolo ${ }^{1}$
}

Affiliations: ${ }^{1}$ Dept of Clinical and Biological Sciences, University of Turin, San Luigi University Hospital, Turin, Italy. ${ }^{2}$ Dept of Veterinary Sciences, University of Turin, Turin, Italy. ${ }^{3}$ Dept of Pneumology and Laboratory of Cytoimmunopathology of the Heart and Lung, Istituti Clinici Scientifici Maugeri, IRCCS, Veruno, Italy. ${ }^{4}$ Respiratory Function and Sleep Laboratory, San Luigi University Hospital, Turin, Italy.

Correspondence: Fabio L.M. Ricciardolo, Dept of Clinical and Biological Sciences, University of Torino, San Luigi University Hospital, Regione Gonzole 10, 10043 Orbassano, Turin, Italy.

E-mail: fabioluigimassimo.ricciardolodunito.it

@ERSpublications

Asthma with high bronchial neutrophilia is associated with increased serum IgE, perennial allergy and IL-17 expression combined with $\mathrm{CD}^{+}$cells. This new endotype is clinically associated with exacerbation rate in the previous year and OCS dependence. http://bit.ly/2ZqboJ2

Cite this article as: Bullone $\mathrm{M}$, Carriero V, Bertolini F, et al. Elevated serum IgE, oral corticosteroid dependence and IL-17/22 expression in highly neutrophilic asthma. Eur Respir J 2019; 54: 1900068 [https:// doi.org/10.1183/13993003.00068-2019].

ABSTRACT Information on the clinical traits associated with bronchial neutrophilia in asthma is scant, preventing its recognition and adequate treatment. We aimed to assess the clinical, functional and biological features of neutrophilic asthma and identify possible predictors of bronchial neutrophilia.

The inflammatory phenotype of 70 mild-to-severe asthma patients was studied cross-sectionally based on the eosinophilic/neutrophilic counts in their bronchial lamina propria. Patients were classified as neutrophilic or non-neutrophilic. Neutrophilic asthma patients (neutrophil count cut-off: 47.17 neutrophils $\cdot \mathrm{mm}^{-2}$; range: 47.17-198.11 neutrophils $\cdot \mathrm{mm}^{-2}$; median: 94.34 neutrophils $\cdot \mathrm{mm}^{-2}$ ) were further classified as high $\left(\geqslant 94.34\right.$ neutrophils $\left.\cdot \mathrm{mm}^{-2}\right)$ or intermediate $\left(47.17-<94.34\right.$ neutrophils $\left.\cdot \mathrm{mm}^{-2}\right)$. The effect of smoking $\geqslant 10$ pack-years was also assessed.

Neutrophilic asthma patients ( $\mathrm{n}=38 ; 36$ mixed eosinophilic/neutrophilic) had greater disease severity, functional residual capacity, inhaled corticosteroid (ICS) dose and exacerbations, and lower forced vital capacity (FVC) \% pred and forced expiratory volume in $1 \mathrm{~s}\left(\mathrm{FEV}_{1}\right)$ reversibility than non-neutrophilic asthma patients ( $\mathrm{n}=32 ; 28$ eosinophilic and four paucigranulocytic). Neutrophilic asthma patients had similar eosinophil counts, increased bronchial $\mathrm{CD}^{+}$, interleukin (IL)-17-F $\mathrm{F}^{+}$and IL-22 ${ }^{+}$cells, and decreased mast cells compared with non-neutrophilic asthma patients. $\mathrm{FEV}_{1}$ and $\mathrm{FVC}$ reversibility were independent predictors of bronchial neutrophilia in our cohort. High neutrophilic patients ( $\mathrm{n}=21)$ had increased serum IgE levels, sensitivity to perennial allergens, exacerbation rate, oral corticosteroid dependence, and $\mathrm{CD}^{+}$and $\mathrm{IL}-17 \mathrm{~F}^{+}$cells in their bronchial mucosa. Excluding smokers revealed increased IL- $17 \mathrm{~A}^{+}$and IL-22 $2^{+}$cells in highly neutrophilic patients.

We provide new evidence linking the presence of high bronchial neutrophilia in asthma to an adaptive immune response associated with allergy (IgE) and IL-17/22 cytokine expression. High bronchial neutrophilia may discriminate a new endotype of asthma. Further research is warranted on the relationship between bronchoreversibility and bronchial neutrophilia.

This article has supplementary material available from erj.ersjournals.com

Received: 10 Jan 2019 | Accepted after revision: 29 July 2019

Copyright OERS 2019 


\section{Introduction}

Asthma is a complex heterogeneous disorder characterised by a variety of cellular and molecular inflammatory mechanisms. Neutrophilia in asthma is associated with ageing and oxidative stress, increased disease severity and exacerbations, non-atopy, cigarette smoking exposure, persistent airflow limitation, reduced reversibility, steroid insensitivity, and comorbidities such as rhinosinusitis, sleep disturbances and gastro-oesophageal reflux disease [1].

In spite of the growing interest in neutrophilic inflammation, asthma is still dichotomously defined as eosinophilic or non-eosinophilic [2]. This classification disregards the fact that neutrophilic inflammation may coexist with eosinophilic inflammation in a subset of patients displaying the so-called mixed inflammatory phenotype [3]. This phenotype, at least when observed in sputum samples, is characterised by greater severity $[4,5]$. Sputum neutrophilia is found in up to $28 \%$ of patients [6], while only $3-8 \%$ have mixed granulocytic inflammation [6-8]. Data from bronchial biopsy samples [9], however, suggest a different inflammatory phenotype distribution, but whether bronchial neutrophilia has any significance in asthma remains unclear.

The paucity of information on neutrophilic asthma is partly due to the lack of biomarkers of bronchial/ sputum neutrophilia recognised for use in clinical settings [1], which slows down the development of new biomarkers for this phenotype of the disease. Hence, identifying reliable markers of bronchial neutrophilia in asthma is essential. Age and functional residual capacity (FRC) were previously identified as significant predictors of sputum neutrophilia $>76 \%$ [7]. Recent evidence suggests that assessing airway inflammation based on sputum may be misleading, especially in paucigranulocytic asthma [10]. For this reason, we chose to define asthma inflammatory phenotypes based on bronchial inflammation using biopsy samples. This study was set up 1) to assess the clinical, functional and biological differences in mild-to-severe asthma patients with versus without bronchial neutrophilia, based on cut-offs previously shown to differentiate patients versus controls [11], and 2) to identify significant predictors of bronchial neutrophilia in our sample. Moreover, we aimed to identify among the parameters studied those that were related to (or influenced by) the magnitude of bronchial neutrophilia. To do this, neutrophilic patients were divided into two groups using the median value of cell counts as cut-off $\left(\geqslant 94.34\right.$ neutrophils $\left.\cdot \mathrm{mm}^{-2}\right)$. As this cut-off was greater than those previously related to severe $\left(\geqslant 50.31\right.$ neutrophils $\left.\cdot \mathrm{mm}^{-2}\right)$ and exacerbation-prone asthma (two or more exacerbations per year; $\geqslant 78.62$ neutrophils $\cdot \mathrm{mm}^{-2}$ ) in a subset of the patients included in the current study [11], we expect our results on highly neutrophilic patients to help expand our knowledge on the pathobiology (endo/phenotyping) of neutrophilic exacerbation-prone asthma.

Of note, this study addresses novel questions with respect to a previous study published by our group [11]. Specifically: 1) it investigates the implications of high bronchial neutrophilia in asthma, 2) it analyses the effects of smoking on the neutrophilic component of bronchial inflammation and 3) it provides new data in support of a subendotype of neutrophilic asthma characterised by an intimate relationship between the neutrophils-allergy-interleukin (IL)-17 pathway, an immune pathological pathway associated with exacerbation-prone asthma.

\section{Methods}

Patients were recruited as previously described [11]. More details are available in the supplementary material. The asthma inflammatory phenotypes were initially described using bronchial eosinophil $\left(12.45\right.$ cells $\left.\cdot \mathrm{mm}^{-2}\right)$ and neutrophil $\left(47.17 \mathrm{cells} \cdot \mathrm{mm}^{-2}\right)$ cut-offs previously shown to differentiate controls from asthma patients [11]. Patients were then classified as neutrophilic or non-neutrophilic $(\geqslant 47.17$ versus $<47.17$ neutrophils $\cdot \mathrm{mm}^{-2}$ ), irrespective of the concomitant bronchial eosinophil content. Lastly, neutrophilic patients were described as high neutrophilic $\left(\geqslant 94.34\right.$ neutrophils $\left.\cdot \mathrm{mm}^{-2}\right)$ or intermediate neutrophilic $\left(47.17-<94.34\right.$ neutrophils $\cdot \mathrm{mm}^{-2}$ ), based on the median neutrophil cell count in this group. Figure 1 details the study design.

\section{Statistical analysis}

Differences among the four inflammatory phenotypes described were assessed using the Kruskal-Wallis test and Dunn's post-test (mixed inflammatory phenotype used as control). Differences in neutrophilic versus non-neutrophilic and high versus intermediate neutrophilic asthma were studied with the MannWhitney or unpaired t-test, depending on data distribution, including and excluding smokers $(\geqslant 10$ pack-years). We used the Chi-squared test and Fisher's exact test to compare proportions between groups. The effects of neutrophilia and disease severity were studied with two-way ANOVA and Śídák's multiple comparison post-tests. Correlations were performed using Pearson or Spearman tests, depending on data distribution (assessed with the D'Agostino-Pearson omnibus normality test). 


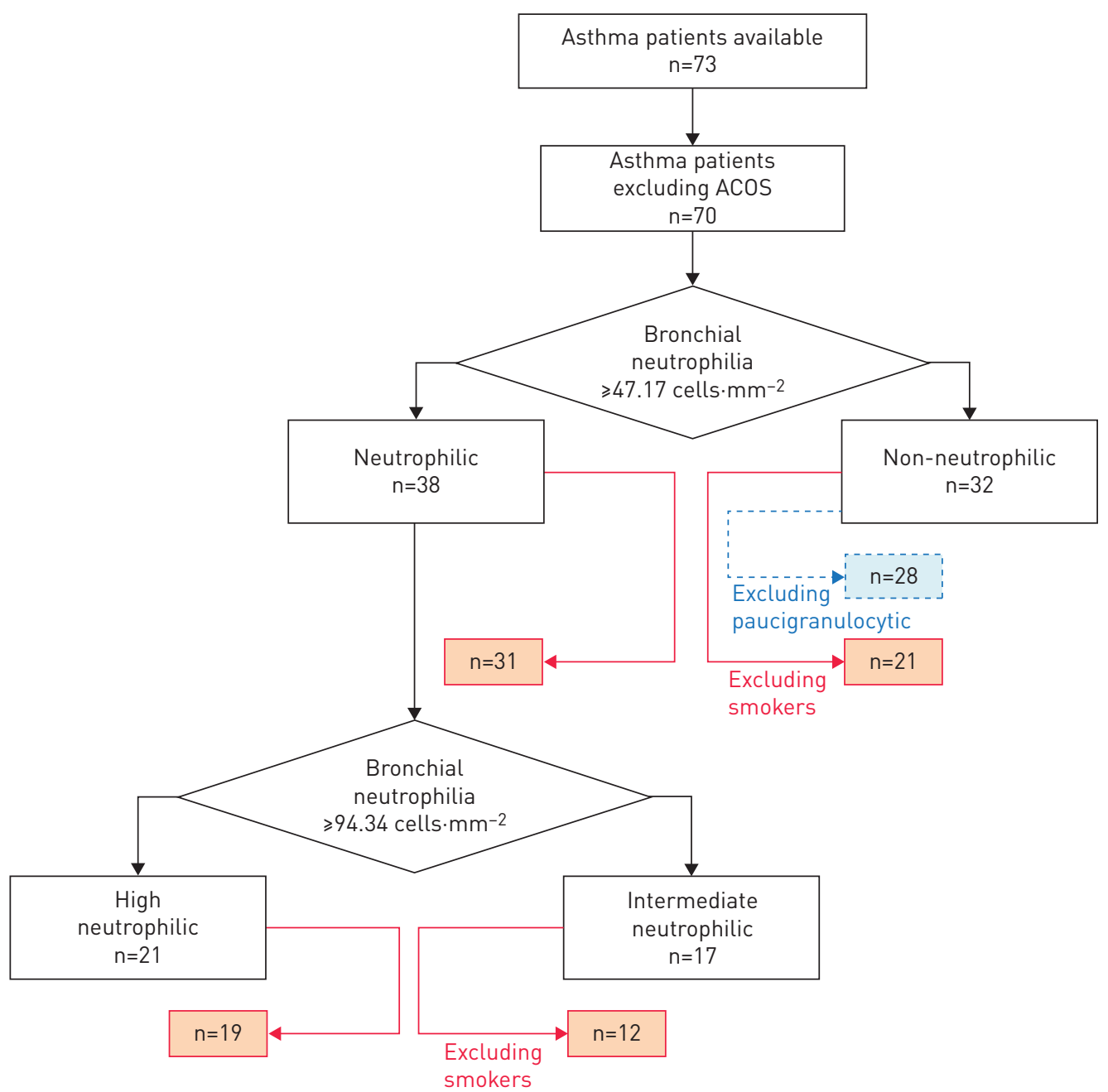

FIGURE 1 Study design. ACOS: asthma-chronic obstructive pulmonary disease overlap syndrome.

Logistic regression analysis on all asthma patients assessed the association between the binary outcome bronchial neutrophilia (yes/no; cut-off: $\geqslant 47.17$ cells $\cdot \mathrm{mm}^{-2}$ ) and the predictors: inhaled corticosteroid (ICS) dose, smoking history ( $\geqslant 10$ versus $<10$ pack-years), forced vital capacity (FVC) $\%$ pred, forced expiratory volume in $1 \mathrm{~s}\left(\mathrm{FEV}_{1}\right) / \mathrm{FVC}, \Delta \mathrm{FEV}_{1}$ and $\triangle \mathrm{FVC}$. Statistical significance was set at $\mathrm{p} \leqslant 0.05$. More details are reported in the supplementary material.

\section{Results}

Inflammatory phenotypes

Clinical details and bronchial inflammatory cell counts of the 70 patients studied are presented in supplementary tables S1 and S2. Our sample was composed mainly of mixed granulocytic ( $\mathrm{n}=36(51 \%))$ and isolated eosinophilic $(\mathrm{n}=28(40 \%))$ asthma patients, with a small minority of isolated neutrophilic $(\mathrm{n}=2(3 \%))$ and paucigranulocytic $(\mathrm{n}=4(6 \%))$ patients (figure 2$)$.

\section{Neutrophilic asthma}

Neutrophilic (isolated neutrophilic and mixed granulocytic) asthma was observed in 38 out of 70 (54\%) patients, most of whom (36 out of $38(95 \%)$ ) had concomitant bronchial eosinophilia. Clinical/functional and biological differences between neutrophilic and non-neutrophilic asthma are reported in tables 1 and 2. Overall, neutrophilic asthma was associated with an increased frequency of severe disease $(p=0.03)$, more exacerbations in the previous 12 months $(\mathrm{p}=0.02)$, a decreased $\mathrm{FEV}_{1}$ response to bronchodilators $\left(\Delta \mathrm{FEV}_{1}\right)(\mathrm{p}=0.004)$ and functional markers of airway closure/air trapping (decreased $\mathrm{FVC} \% \mathrm{pred}, \mathrm{p}=0.03$; increased FRC \% pred, $\mathrm{p}=0.04)$ compared with non-neutrophilic asthma. ICS dose was more than double that in non-neutrophilic asthma $(\mathrm{p}=0.004)$ and the frequency of patients on oral corticosteroid (OCS) for $\geqslant 6$ months per year tended to be higher in the presence of bronchial neutrophilia $(p=0.06)$. Stratifying 

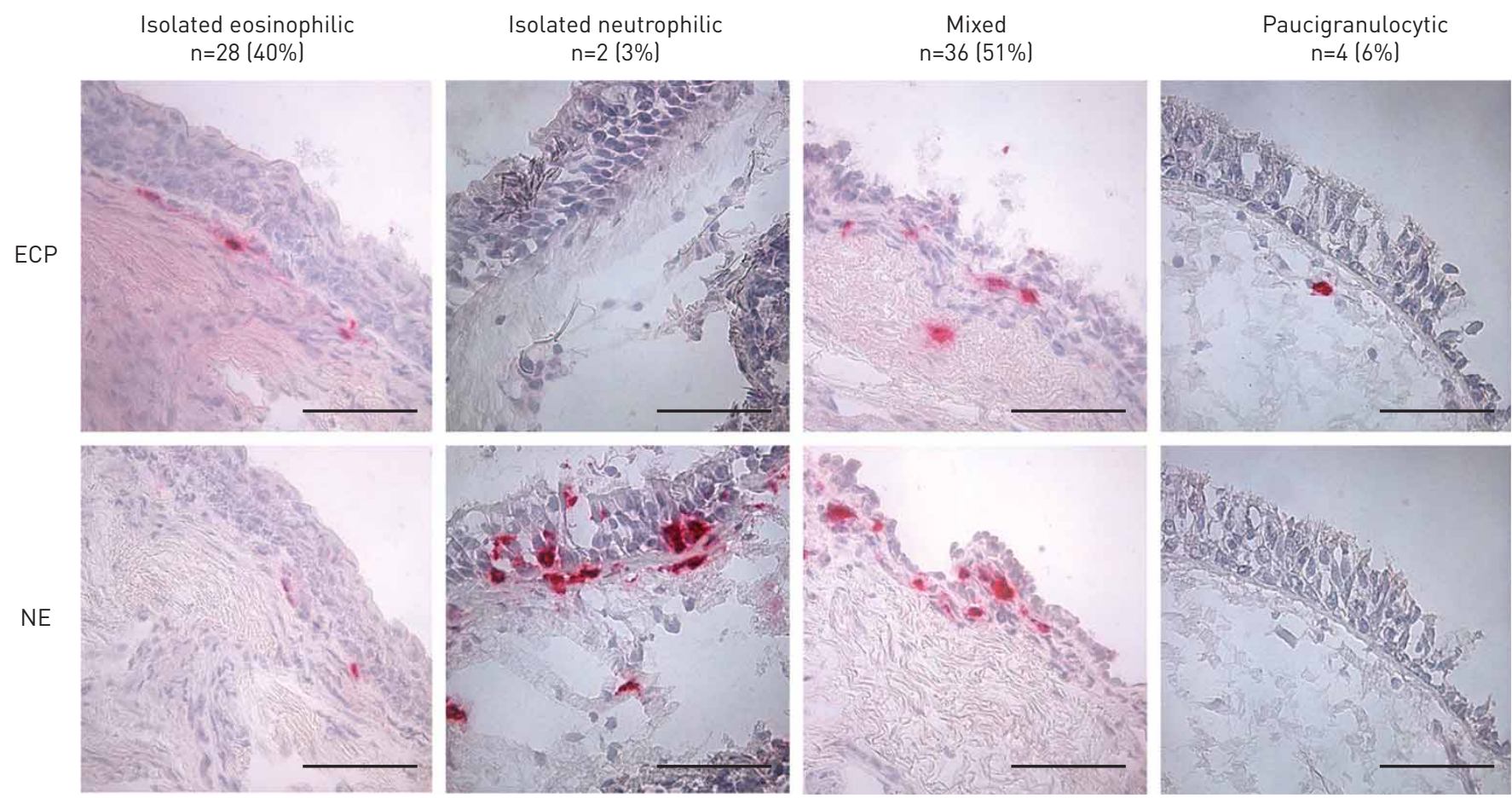

FIGURE 2 Distribution and representative images of the asthma inflammatory phenotypes studied, showing cells staining positive for eosinophil cationic protein (ECP; upper panels) and neutrophil elastase (NE; lower panels). Scale bar: $50 \mu \mathrm{m}$.

patients based on both bronchial neutrophilia and disease severity showed that ICS dose was associated with disease severity $(\mathrm{p}<0.0001)$ and with bronchial neutrophilia $(\mathrm{p}=0.04)$ (figure $3 \mathrm{c})$. The number of patients on OCS for $\geqslant 6$ months per year was significantly higher among severe versus mild neutrophilic asthma patients (7 out of 23 versus 0 out of $15 ; \mathrm{p}=0.03$ ), but not in neutrophilic versus non-neutrophilic severe asthma patients ( 7 out of 23 versus 1 out of $11 ; \mathrm{p}=0.22$ ). We found a significant correlation between ICS dose and bronchial neutrophilia in all asthma patients (supplementary figure S1).

According to this subanalysis, FVC reversibility $(\Delta \mathrm{FVC})$ was greater in neutrophilic than non-neutrophilic patients in severe asthma patients only $(\mathrm{p}=0.008)$ (figure $3 \mathrm{a})$, in spite of similar values of airway obstruction $\left(\mathrm{FEV}_{1} \%\right.$ pred and $\mathrm{FEV}_{1} / \mathrm{FVC}$ ) (supplementary figure S2). Values of $\Delta \mathrm{FVC}>315 \mathrm{~mL}$ discriminated severe (but not mild) neutrophilic versus non-neutrophilic patients with $70 \%$ sensitivity and $78 \%$ specificity (area under the curve $0.72 ; \mathrm{p}=0.05 ; 3.13$ likelihood ratio) (figure $3 \mathrm{~g}$ ). In contrast, $\Delta \mathrm{FEV}_{1}$ $<340 \mathrm{~mL}$ discriminated only mild neutrophilic versus non-neutrophilic patients with $77 \%$ sensitivity and $76 \%$ specificity (area under the curve $0.80 ; \mathrm{p}=0.005 ; 3.27$ likelihood ratio) (figure $3 \mathrm{~h}$ ). In light of the $\Delta \mathrm{FVC}$ results, we investigated the presence and degree of air trapping in our cohort in more depth. Severe neutrophilic asthma patients had the highest numerical value of residual volume (RV) \% pred and the highest proportion of air trappers (defined as patients with $\mathrm{RV} \geqslant 130 \%$ predicted) (figure $3 \mathrm{~d}$ ), but differences were not statistically significant. When mild and severe neutrophilic patients were pooled, the proportion of air trappers was similar to that observed in non-neutrophilic patients ( $p=0.6)$ (figure $3 e$ ). Among air trappers, those with bronchial neutrophilia tended to have higher values of RV \% pred $(\mathrm{p}=0.08$ ) (figure $3 \mathrm{f}$ ). Although RV/total lung capacity (TLC) did not reach statistical significance, it tended to be higher in neutrophilic than in non-neutrophilic patients $(\mathrm{p}=0.06)$.

In their bronchial lamina propria, neutrophilic patients had more CD8 ${ }^{+}, \mathrm{IL}-17-\mathrm{F}^{+}$and $\mathrm{IL}-22^{+}$cells and fewer mast cells compared with non-neutrophilic patients. A trend was also evident towards higher numbers of $\mathrm{CD}^{+}$and IL-17A $\mathrm{A}^{+}$cells in neutrophilic compared with non-neutrophilic patients (table 2). Excluding paucigranulocytic patients from the non-neutrophilic group left the medians of all parameters unchanged (data not shown).

The proportion of smokers was not different between neutrophilic and non-neutrophilic patients $(\mathrm{p}=0.17)$. However, as bronchial neutrophilia has been strongly linked to smoking [12], we repeated the analysis excluding current and ex-smokers ( $\geqslant 10$ pack-years). Briefly, in this reduced sample $(n=52 ; 31$ neutrophilic and 21 non-neutrophilic), we confirmed that neutrophilic patients were more frequently affected by severe asthma $(\mathrm{p}=0.002)$, with increased airflow limitation $(\mathrm{p}=0.02)$, airway closure/air trapping $(\mathrm{p}=0.01)$, 
TABLE 1 Clinical and functional parameters of neutrophilic versus non-neutrophilic asthma patients (smokers included)

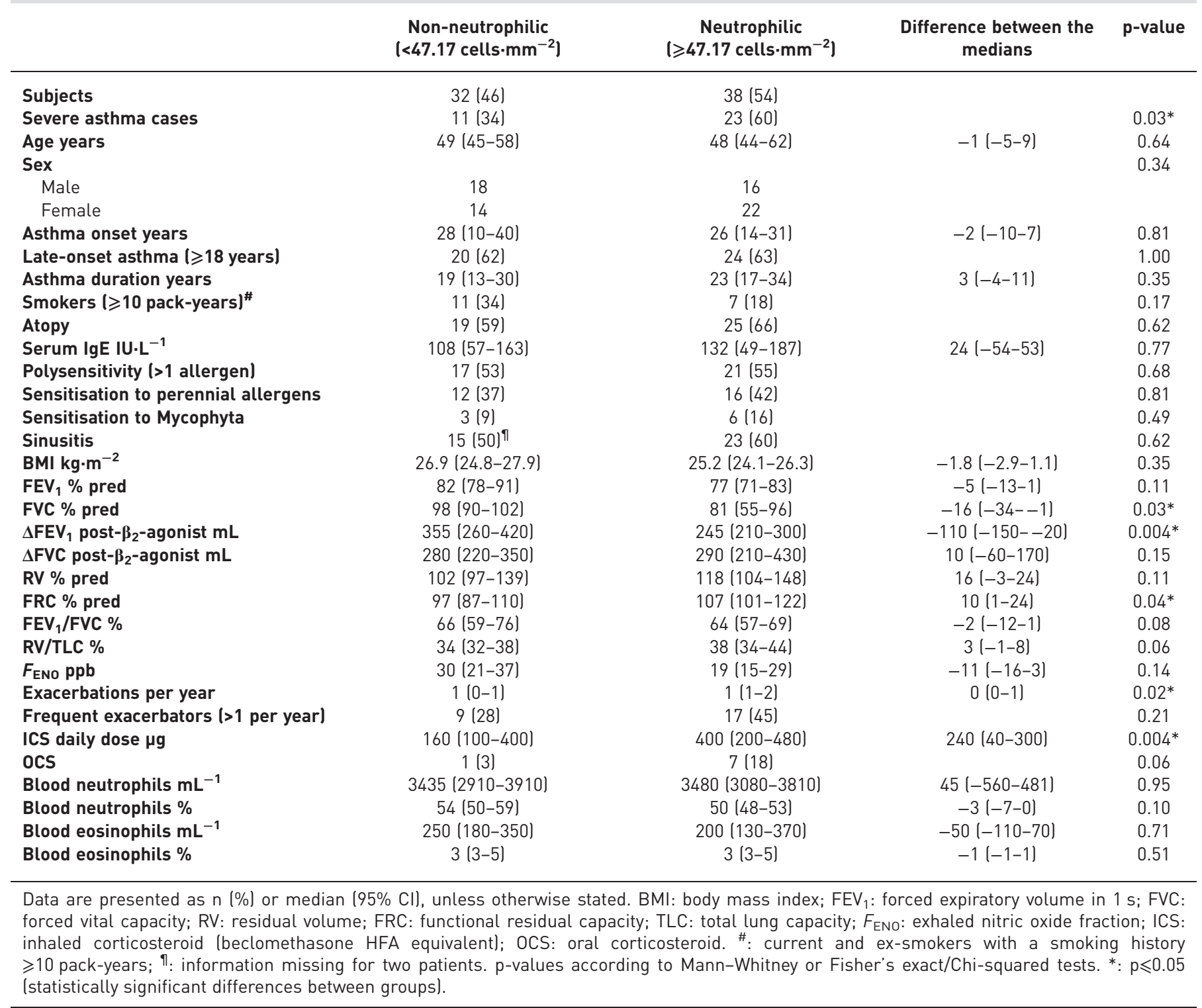

decreased $\Delta \mathrm{FEV}_{1}(\mathrm{p}=0.0007)$ and higher ICS dose $(\mathrm{p}=0.0003)$. Instead, the differences previously observed between neutrophilic and non-neutrophilic asthma concerning number of exacerbations in the previous year, FRC $\%$ pred and number of IL- $22^{+}$cells in the bronchial submucosal were lost, but the number of $\mathrm{CD}^{+}$cells in the lamina propria became significantly greater in neutrophilic asthma $(\mathrm{p}=0.01)$ (supplementary tables S3 andS4).

\section{Highly neutrophilic asthma}

Among neutrophilic asthma patients, there were 21 out of 38 (55\%) with high bronchial neutrophilia (figure $4 \mathrm{a}-\mathrm{c}$ ). Clinical and functional parameters of high versus intermediate neutrophilic asthma patients are reported in supplementary table S5. Asthma patients with high bronchial neutrophilia had increased serum $\operatorname{IgE}(\mathrm{p}=0.02)$, greater sensitisation to perennial allergens $(\mathrm{p}=0.05)$, more frequent exacerbations $(p=0.001)$ and more patients were on OCS for $\geqslant 6$ months per year $(p=0.01)$ compared with those with intermediate bronchial neutrophilia (figure $4 \mathrm{~d}-\mathrm{g}$ ). The bronchial eosinophil count was similar in the two groups ( $\mathrm{p}=0.86$ ) (supplementary table S6). Asthma patients with high bronchial neutrophilia displayed a 3 -fold increase in the number of $\mathrm{IL}-17 \mathrm{~F}^{+}$cells in their submucosa $(\mathrm{p}=0.0001)$ and a greater number of $\mathrm{CD}^{+}$cells $(\mathrm{p}=0.03)$ (supplementary table S6) compared with those with intermediate bronchial neutrophilia. 
TABLE 2 Inflammatory and biological parameters of neutrophilic versus non-neutrophilic asthma patients (smokers included)

\begin{tabular}{|c|c|c|c|c|}
\hline & $\begin{array}{l}\text { Non-neutrophilic } \\
{\left[<47.17 \text { cells } \cdot \mathrm{mm}^{-2} \text { ) }\right.}\end{array}$ & $\begin{array}{c}\text { Neutrophilic } \\
\left(\geqslant 47.17 \text { cells } \cdot \mathrm{mm}^{-2}\right\rfloor\end{array}$ & $\begin{array}{l}\text { Difference } \\
\text { between the } \\
\text { medians }\end{array}$ & p-value \\
\hline Subjects & $32(46)$ & $38(54)$ & & \\
\hline $\begin{array}{l}\text { Cell counts } \mathrm{mm}^{-2} \\
\text { lamina propria }\end{array}$ & $24(19-42)$ & $38(24-44)$ & $13(-5-16)$ & 0.25 \\
\hline $\mathrm{ECP}^{+}$ & $24(19-42)$ & $38(24-44)$ & $13(-5-16)$ & 0.25 \\
\hline $\mathrm{NE}^{+}$ & 24 (19-33) & $94(80-113)$ & $71(57-85)$ & $<0.0001 *$ \\
\hline $\mathrm{CD}^{+}{ }^{+}$ & $19(9-28)$ & $28(19-35)$ & $9(0-19)$ & 0.07 \\
\hline $\mathrm{CD}^{+}$ & $13(5-24)$ & $23(14-28)$ & $10(1-15)$ & $0.02 *$ \\
\hline $\mathrm{CD}_{68^{+}}$ & $260(159-374)$ & 209 (163-297) & $-51(-131-71)$ & 0.55 \\
\hline Tryptase $^{+}$ & 74 (44-130) & $29(14-87)$ & $-45(-60-0)$ & $0.04^{*}$ \\
\hline IL-17A & $9(5-19)$ & $19(11-24)$ & $9(0-13)$ & 0.06 \\
\hline IL-17F+ & $13(8-24)$ & 21 (13-33) & $8(1-17)$ & $0.03^{*}$ \\
\hline $\mathrm{IL}-21^{+}$ & $17(9-24)$ & $19(12-27)$ & $1(-5-9)$ & 0.57 \\
\hline $\mathrm{IL}^{-22^{+}}$ & $13(9-25)$ & $19(13-32)$ & $6(0-15)$ & $0.03^{*}$ \\
\hline $\mathrm{IL}-23^{+}$ & $16(9-24)$ & $14(9-19)$ & $-2(-6-7)$ & 0.72 \\
\hline
\end{tabular}

After excluding neutrophilic patients with a smoking history, asthma patients with high bronchial neutrophilia still had higher serum $\operatorname{IgE}$ levels $(p=0.01)$ and more frequent exacerbations $(p=0.006)$ than asthma patients with intermediate neutrophilia. Sensitivity to perennial allergens and number of patients on OCS still tended to be higher in high versus intermediate neutrophilic patients $(\mathrm{p}=0.06)$. Excluding smokers confirmed the increased number of $\mathrm{CD}^{+}$and $\mathrm{IL}-17 \mathrm{~F}^{+}$cells in high bronchial neutrophilia (figure $5 \mathrm{a}-\mathrm{c}$ and $\mathrm{g}-\mathrm{i}$ ), and also revealed an increased number of IL-17A $\mathrm{A}^{+}$and $\mathrm{IL}-22^{+}$cells in this group (figure $5 \mathrm{~d}-\mathrm{f}$ and $\mathrm{j}-\mathrm{l}$ ).

\section{Correlations}

Among all neutrophilic patients, significant correlations were observed between bronchial neutrophil counts and both the number of exacerbations per year and serum IgE levels, but not ICS dose (figure 6a-c). Bronchial neutrophilia also correlated with $\mathrm{CD} 4^{+}, \mathrm{IL}_{-}-22^{+}$and $\mathrm{IL}-17 \mathrm{~F}^{+}$cells (figure $6 \mathrm{~d}-\mathrm{f}$ ), and negatively correlated with bronchial mast cell counts $\left(r_{s}=-0.27 ; p=0.04\right)$. A negative correlation was also observed between bronchial mast cell and IL-17A ${ }^{+}$cell counts $\left(r_{s}=-0.34 ; p=0.01\right)$ (data not shown). Significant correlations were also observed between IgE and IL-17F $\mathrm{F}^{+}$cells (figure $6 \mathrm{~g}$ ) and IgE and blood eosinophilia expressed both as absolute cell count and as percentage (figure $6 \mathrm{~h}$ and i).

\section{Predictors of bronchial neutrophilia}

Lung function reversibility parameters were the only predictors associated with bronchial neutrophilia $\left(\geqslant 47.17\right.$ cells $\left.\cdot \mathrm{mm}^{-2}\right)$ among those studied (supplementary figure S3). In detail, compared with patients with $\Delta \mathrm{FEV}_{1}<220 \mathrm{~mL}$ (first IQR), those with values $\geqslant 280 \mathrm{~mL}$ (third and fourth IQR) had a significantly lower probability of having bronchial neutrophilia $(\mathrm{p}=0.01)$. Concerning $\triangle F V C$, the probability of having bronchial neutrophilia was similar in patients with values $<210 \mathrm{~mL}$ (first IQR) or $210-305 \mathrm{~mL}$ (second $\mathrm{IQR}$ ). Instead, patients with values $\geqslant 450 \mathrm{~mL}$ (fourth $\mathrm{IQR}$ ) had a significantly greater probability of having bronchial neutrophilia compared with those with $210-305 \mathrm{~mL}$ (second IQR; $\mathrm{p}=0.006$ ) (supplementary table S7). Further details are provided in the supplementary material.

\section{Discussion}

Asthma inflammatory phenotypes are commonly defined on the basis of sputum cell counts. About 20$30 \%$ of adult asthma patients of all severities have sputum neutrophilia $[6,7]$, mostly in the absence of concomitant eosinophilia. The proportion of patients with sputum neutrophilia increases in severe asthma [5]. In our study on bronchial biopsies, we observed bronchial neutrophilia in 54\% of mild-to-severe patients and this percentage further rose to $68 \%$ when only severe patients were considered. Excluding smokers, $60 \%$ of our patients were neutrophilic, indicating that neutrophils are key players in asthma pathogenesis, and this merits further investigation. Whether sputum cell counts reflect bronchial 
a)
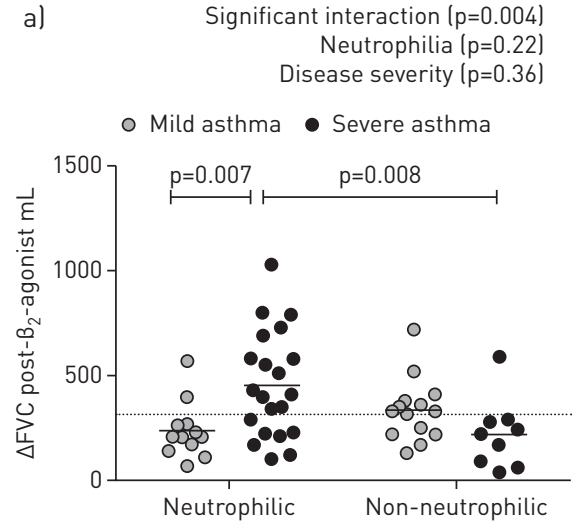

d)

Significant disease severity $(\mathrm{p}=0.006)$ Neutrophilia $(p=0.29$ Interaction $(p=0.1)$
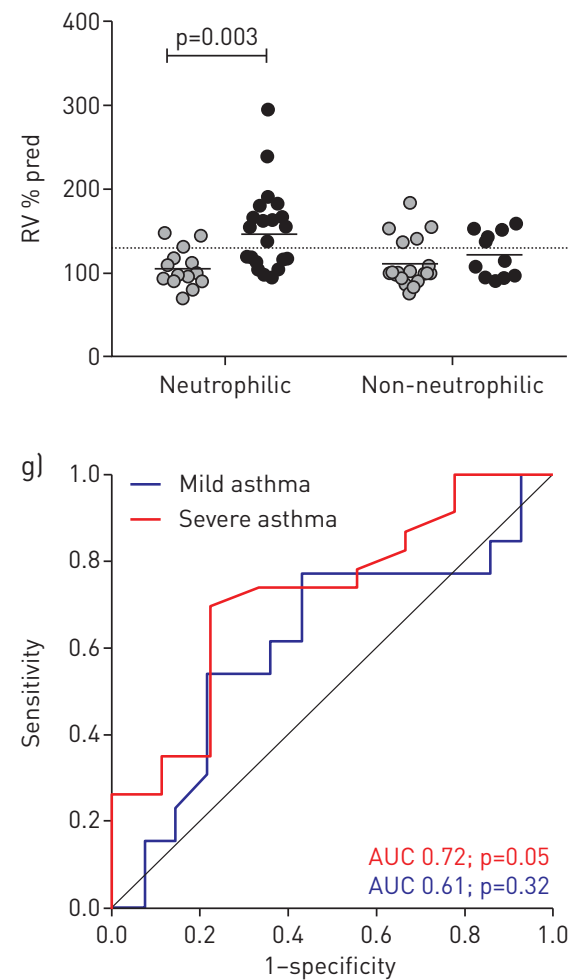

b)
Significant neutrophilia $(p=0.006)$ Interaction $(\mathrm{p}=0.06)$ Disease severity $(p=0.08)$
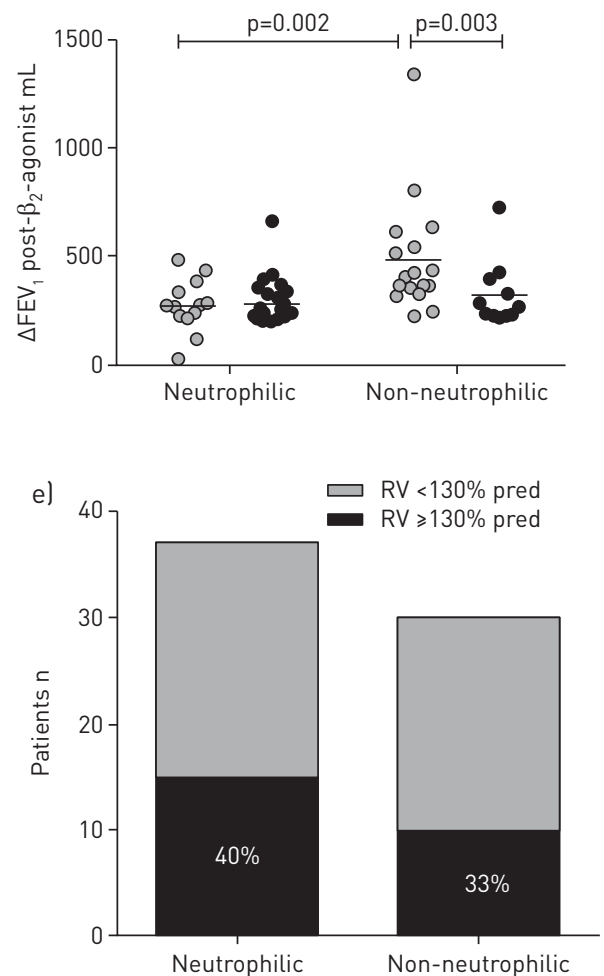

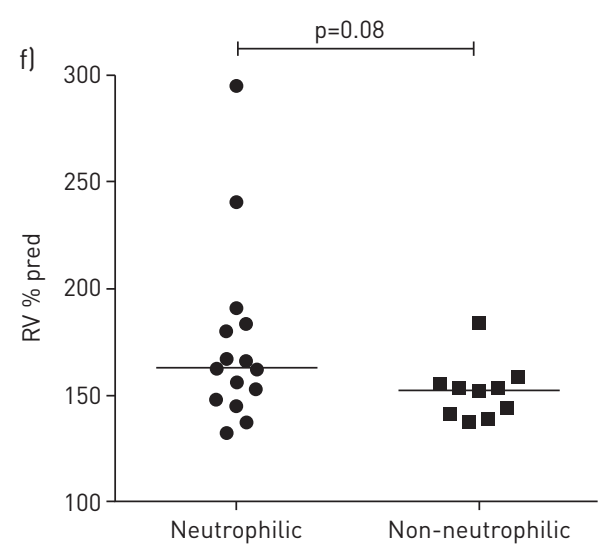

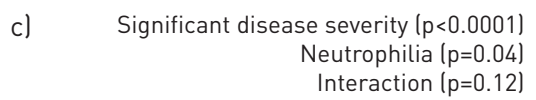

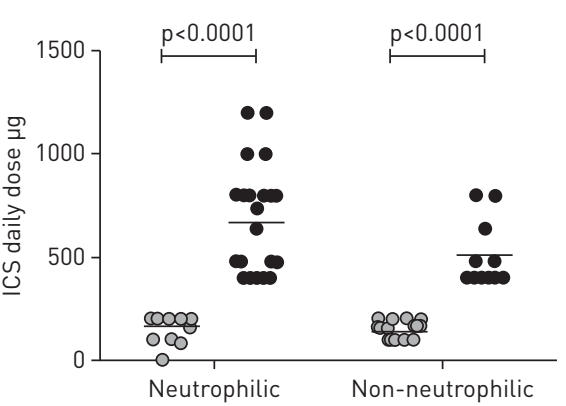

FIGURE 3 Effect of bronchial neutrophilia and disease severity on bronchodilator-induced a) change in forced vital capacity ( $\triangle F V C)$ and b) change in forced expiratory volume in $\left.1 \mathrm{~s}\left(\Delta \mathrm{FEV}_{1}\right), \mathrm{c}\right)$ inhaled corticosteroid (ICS) dose (beclomethasone HFA equivalent), and $\mathrm{d}-\mathrm{f}$ ) basal residual volume (RV) \% pred. In f) only patients with RV $\geqslant 130 \%$ pred (air trappers) were included in the analysis. The dashed lines in (a) and (d) represent the cut-off values for $\triangle \mathrm{FVC}(315 \mathrm{~mL})$ and RV \% pred $(130 \%)$, respectively. Mean values are indicated in the plots. g. h) Receiver operating characteristic curves for optimal cut-off points at which g) $\Delta \mathrm{FVC}$ and $\mathrm{h}) \Delta \mathrm{FEV}_{1}$ discriminate mild or severe asthma patients with bronchial neutrophilia. AUC: area under the curve. Smokers were included.

wall inflammation remains an open question. While it seems to be the case for eosinophils, available data suggest that sputum neutrophilia cannot predict bronchial neutrophilic inflammation $[13,14]$, underlining the need for alternative non-invasive and reliable markers for bronchial neutrophilia.

The paucity of information about the clinical traits associated with bronchial neutrophilia in asthma prevents its recognition, the implementation of adequate treatment and the possibility to improve disease control [1]. Our data reveal that bronchial neutrophilia is associated with severe asthma and characterised by reduced $\mathrm{FEV}_{1}$ reversibility $\left(\Delta \mathrm{FEV}_{1}\right)$, prominent airway closure/air trapping and pulmonary hyperinflation (lower FVC \% pred, higher FRC \% pred, borderline difference noticed also for RV/TLC). Due to the close relationship among smoking, neutrophilia and permanent airway obstruction [12], we 

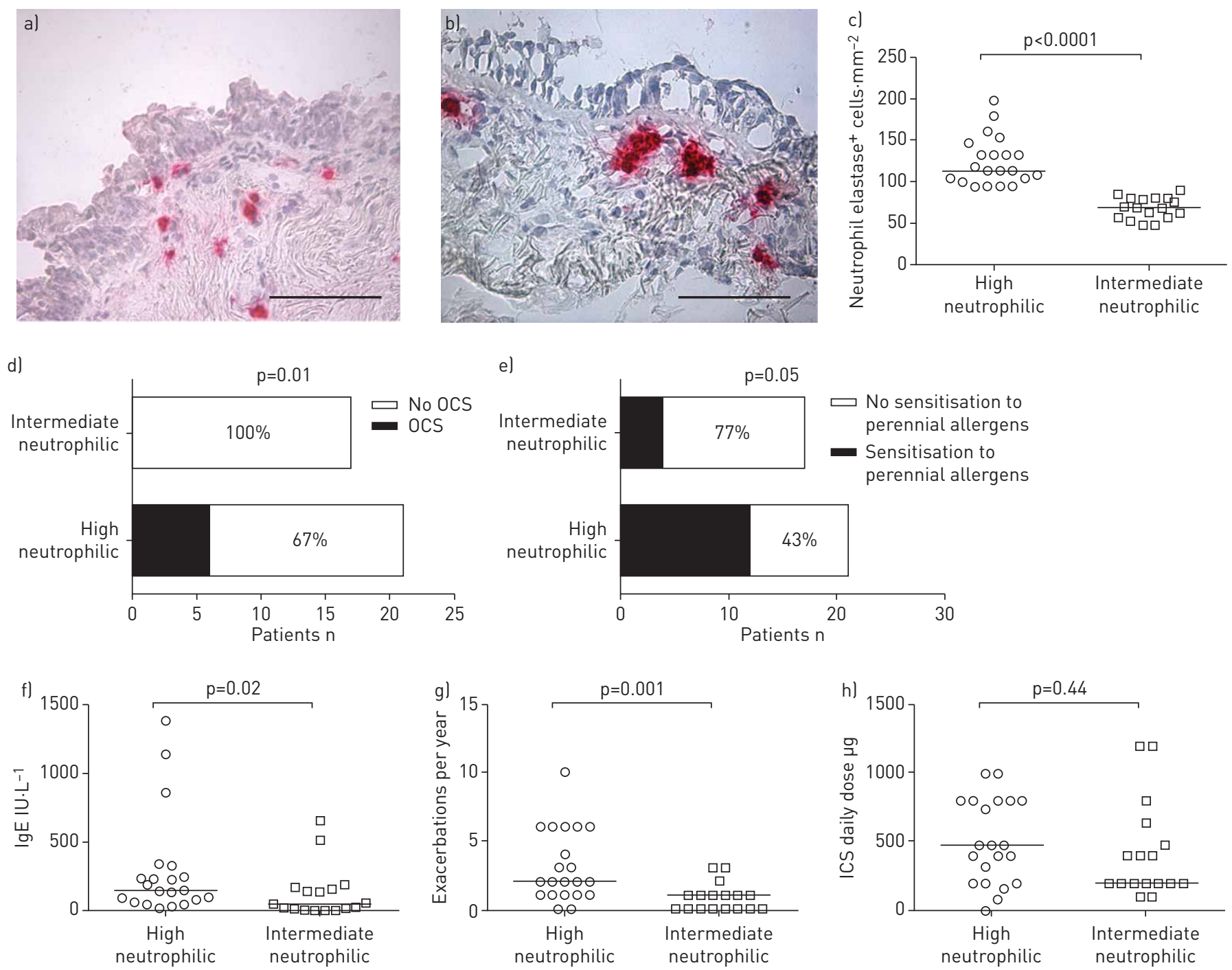

FIGURE $4 \mathrm{a}-\mathrm{c}$ ) Representative images of a) high ( $\geqslant 94.34$ cells $\cdot \mathrm{mm}^{-2}$ ) and b) intermediate (47.17-<94.34 cells $\cdot \mathrm{mm}^{-2}$ ) bronchial neutrophilia, and c) relative counts. Scale bar: $50 \mu \mathrm{m}$. d-h) Clinical parameters associated with high bronchial neutrophilia in asthma patients: d) oral corticosteroid (OCS), e) sensitisation to perennial allergens, f) $\mathrm{lgE}, \mathrm{g}$ ) exacerbations and h) inhaled corticosteroid (ICS) dose (beclomethasone HFA equivalent). Mean values are indicated in the plots.

repeated our analysis excluding smokers. This revealed that pulmonary hyperinflation likely results from smoking (or smoking-related neutrophilia). It confirmed low FVC $\%$ pred and $\triangle \mathrm{FEV}_{1}$ as clinical traits of bronchial neutrophilia, its association with disease severity, and revealed a significantly greater airflow limitation (reduced $\mathrm{FEV}_{1} / \mathrm{FVC}$ ) in non-smoking neutrophilic versus non-neutrophilic patients. Overall, bronchial neutrophilia characterises patients with a more severe smoking-independent form of asthma that affects all levels of the bronchial tree and manifests as enhanced obstruction and reduced reversibility, in agreement with previous studies $[15,16]$. In light of previous data showing asthma severity as a critical determinant of the bronchodilator-induced reversibility response [17], we examined the combined effect of bronchial neutrophilia and disease severity on bronchoreversibility parameters. Our findings show the existence of significant interactions between bronchial neutrophilia and disease severity, with distinct patterns of bronchoreversibility in mild and severe asthma patients. Mild asthma patients have low $\Delta \mathrm{FVC}$ irrespective of their bronchial neutrophilia, likely indicating the absence of air trapping/airway closure, while $\triangle \mathrm{FEV}_{1}$ is reduced only when bronchial neutrophilia is present in this group. Given the similar ICS doses in neutrophilic and non-neutrophilic mild asthma patients (and similar values of $\mathrm{FEV}_{1} \%$ pred and $\mathrm{FEV}_{1} / \mathrm{FVC}$ indicating similar degrees of obstruction), the reduced $\triangle \mathrm{FEV}_{1}$ observed with neutrophilia could reflect irreversible airway remodelling or steroid resistance in this subset of patients. In contrast, severe asthma patients all had low $\triangle \mathrm{FEV}_{1}$, confirming the reduced reversibility of obstruction in these patients, while $\triangle \mathrm{FVC}$ was higher in neutrophilic asthma patients (with similar $\mathrm{FEV}_{1} \%$ pred and $\mathrm{FEV}_{1} / \mathrm{FVC}$ ). On 

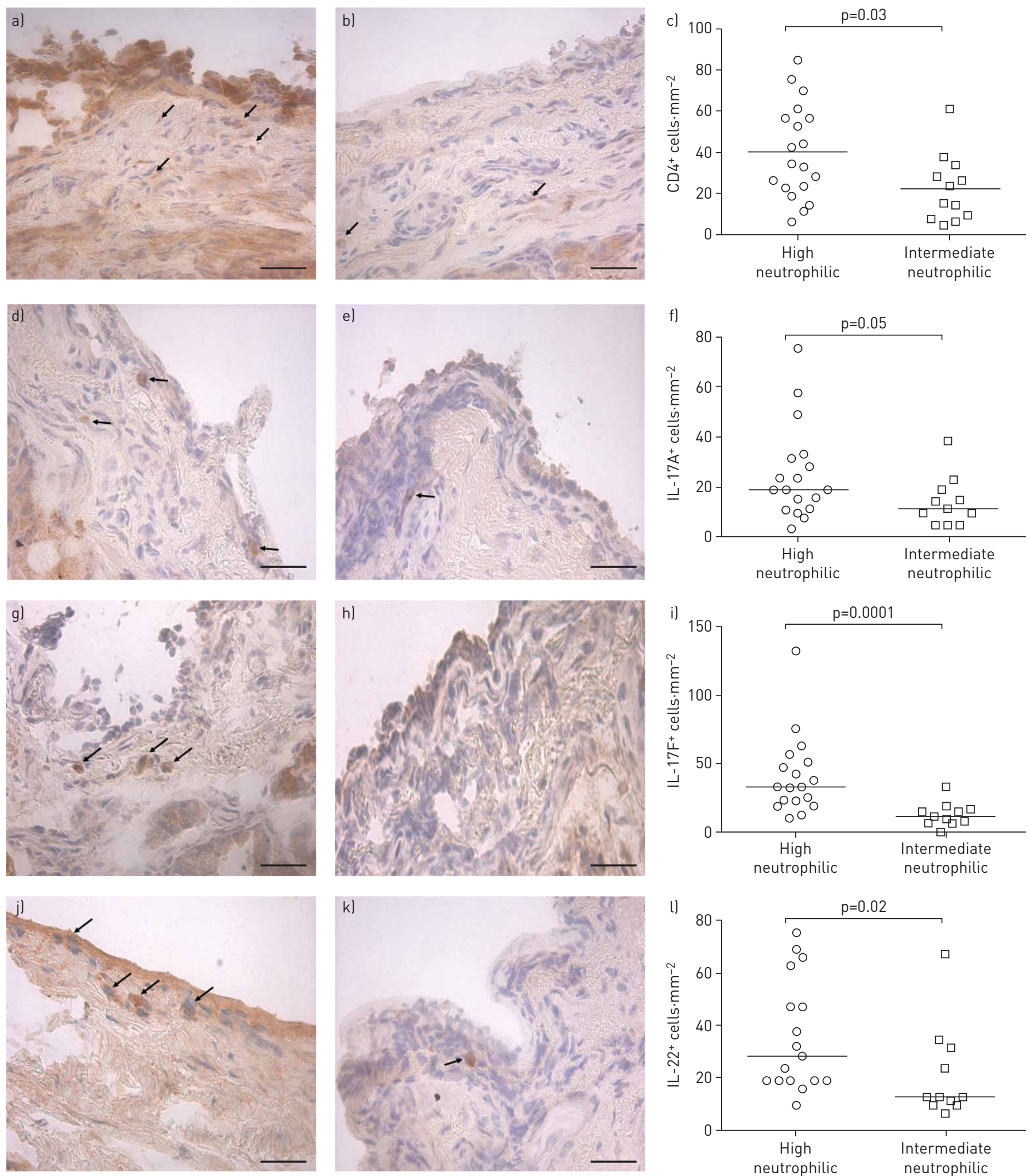

FIGURE 5 T-helper cell Th17 signature in neutrophilic asthma (smokers excluded). IL: interleukin. Representative images and bronchial count values of asthma patients with $a, d, g, j)$ high ( $\geqslant 94.34 \mathrm{cells} \cdot \mathrm{mm}^{-2}$ ) and b, e, h, k) intermediate (47.17-<94.34 cells $\mathrm{mm}^{-2}$ ) neutrophilia, immunostained for a-c) CD4, d-f) IL-17A, g-i) IL-17F and j-l) IL-22. Scale bar: $50 \mu \mathrm{m}$. Mean values are indicated in the plots.

further analysis, $\Delta \mathrm{FEV}_{1}<340 \mathrm{~mL}$ and $\Delta \mathrm{FVC}>315 \mathrm{~mL}$ were identified as significant discriminants of bronchial neutrophilia in mild and severe asthma patients, respectively. This is in line with recent studies reporting that $\triangle \mathrm{FVC}$ increases with the level of airflow obstruction $[18,19]$, and that patients with FVC 

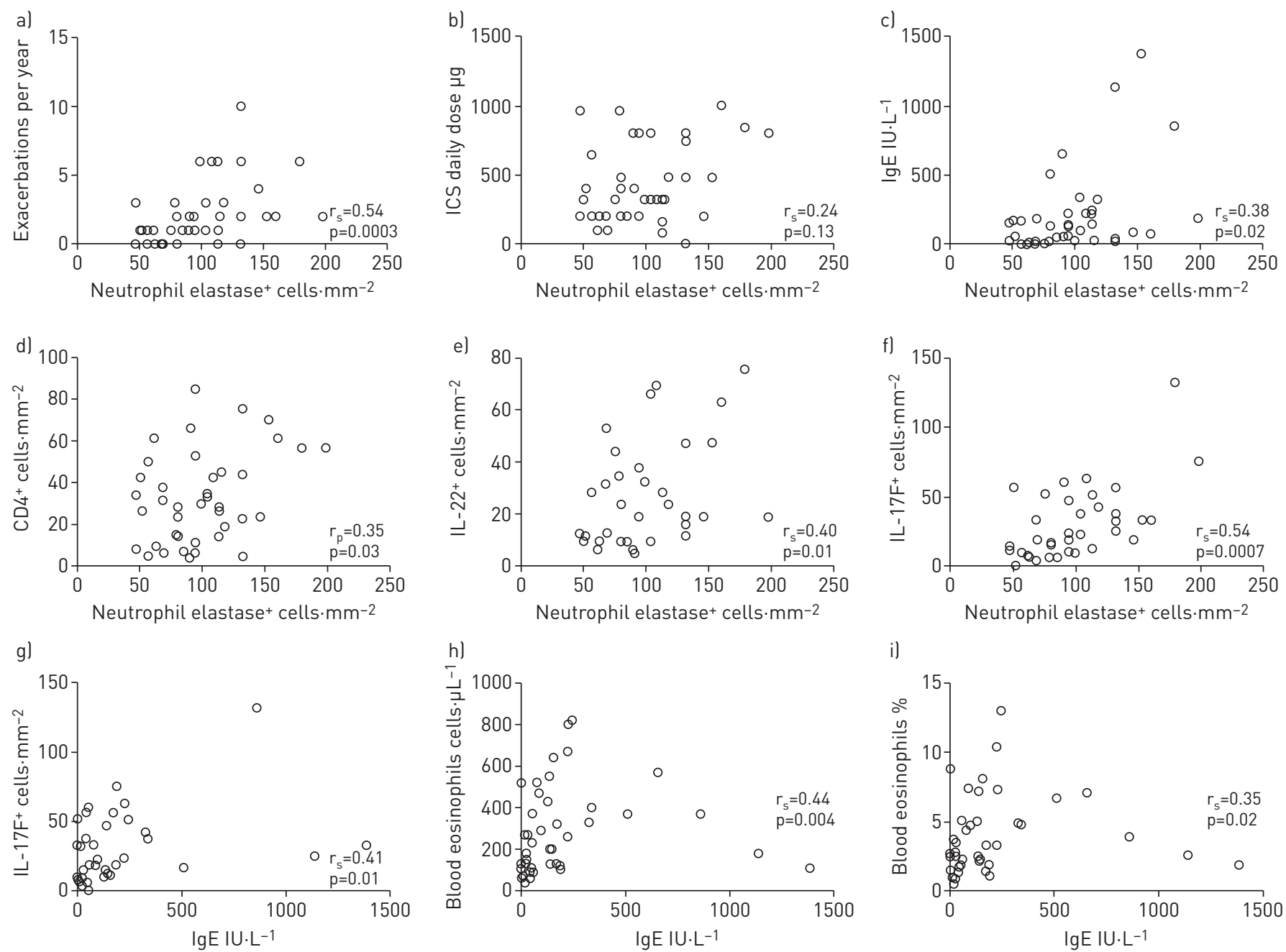

FIGURE 6 a-f) Correlations between bronchial neutrophilia and a) number of exacerbations in the last year, b) inhaled corticosteroid (ICS) dose (beclomethasone HFA equivalent), c) serum IgE levels, d) $\mathrm{CD4}^{+}$, e) interleukin (IL)-22 ${ }^{+}$and f) IL-17F ${ }^{+}$cells in bronchial tissue. g-i) Correlations between serum IgE levels and g) IL-17F cells and $h$, i) blood eosinophilia. Neutrophilic patients only $(n=41)$. The Pearson $\left(r_{p}\right)$ or $S p e a r m a n\left(r_{s}\right)$ correlation coefficient is indicated.

reversibility (either alone or coexisting with $\mathrm{FEV}_{1}$ reversibility) have worse lung function and poorer asthma control than patients with $\mathrm{FEV}_{1}$ reversibility only [20]. Further studies will be needed to verify our findings, but the available evidence suggests that both $\triangle \mathrm{FEV}_{1}$ and $\triangle \mathrm{FVC}$ merit attention during the clinical assessment of asthma patients.

The role of neutrophils in asthma is unclear. Our data suggest that increased bronchial neutrophils alter the lung function, but there is no progressive worsening with further increases. Stepping up from intermediate to high bronchial neutrophilia was instead associated with an increase in serum IgE, sensitisation to perennial allergens, OCS treatment and exacerbations, although mast cell numbers remained unchanged. Patients with high bronchial neutrophilia also had increased airway $\mathrm{CD}^{+}$and IL- $17 \mathrm{~F}^{+}$cells, and values of neutrophil counts, serum IgE, IL- $17 \mathrm{~F}^{+}$and $\mathrm{CD} 4^{+}$cells strongly correlated, indicating a possible role of neutrophils and IL-17 in allergic mechanisms in this disease phenotype, in accordance with a dual T-helper cell Th2/Th17 immune response [21, 22]. Smoke exposure might influence bronchial neutrophils, serum IgE and Th17 cytokine levels [23, 24]. Repeating the analysis excluding smokers, however, confirmed our data, also revealing an increased number of cells expressing IL-17A and IL-22, two Th17 effector cytokines [25], in highly neutrophilic asthma. In this context, previous studies support an interplay of serum IgE and neutrophils in asthma [26], possibly leading to a phenotype characterised by atopy and increased exacerbations [27], in line with our observations. IgE exposure induces passive sensitisation of airway smooth muscle cells, enhancing their contractile response in vitro [28] and possibly favouring exacerbations in vivo. Interestingly, our study also showed a decreased number of mast cells in neutrophilic compared with non-neutrophilic asthma and negative correlations 
between mast cells and neutrophils or IL-17A, suggesting that IL-17-driven neutrophilic inflammation could be associated with a concomitant decrease of mast cell-related inflammation in asthma.

The IL-17 pathway has been associated with severe and neutrophilic asthma, with the frequent exacerbator asthma phenotype $[11,25,29]$, and also with atopic asthma [27, 30]. While Th2 and Th17 are generally viewed as being mutually exclusive immune pathways, significant interactions have been shown between Th17 and Th2 responses, with dual Th2/Th17 T-cells found in blood and bronchoalveolar lavage of stable allergic and severe asthma patients $[21,22]$. Data from a murine model suggest that dual Th2/Th17 T-cells (able to induce IgE secretion from B-cells in vitro) are increased in the lungs during the chronic asthma phase, and may be responsible for disease exacerbation through the recruitment of neutrophils and eosinophils [22]. Accordingly, bronchial neutrophilia was observed concomitantly with bronchial eosinophilia in our patients, except for two cases. The rise in neutrophils and eosinophils was not correlated, however, possibly because bronchial eosinophils decrease following corticosteroid treatment in asthma, while the effect is dampened for neutrophils [31,32]. Alternatively, the corticosteroid-induced increase in neutrophil phagocytic activity [33] might explain the more effective removal of eosinophils from the bronchial tissue. Taken together, our data suggest that in a subset of neutrophilic asthma patients, allergic mechanisms deviate from a classical Th2 to an alternative Th17 or dual Th2/ Th17-mediated immune response and are associated with increased exacerbation frequency. The molecular mechanisms by which this paradigm shift occurs and neutrophils contribute to the allergic response warrant further attention.

Data on the effect of corticosteroids on bronchial neutrophils are discordant. Although the majority of studies on bronchial biopsies show a decrease or a steady state of neutrophils after corticosteroid treatment [32, 34-37], some report an increase [38]. Of note, however, lymphocytes were always decreased or remained unchanged in these studies and in other sputum studies where ICS-induced neutrophilia was observed [39]. In our study, we found a difference in ICS dose between neutrophilic and non-neutrophilic mild-to-severe asthma patients, and a correlation between ICS and bronchial neutrophilia in the whole population studied. We also noted a concomitant increase of bronchial neutrophils and $\mathrm{CD}^{+}$cells, in association with elevated activity of the IL-17 pathway. Moreover, ICS dose was not different between high and intermediate neutrophilic asthma, and did not correlate with the number of neutrophils in high-intermediate neutrophilic asthma. In vitro, IL-17 cytokines induce steroid resistance in peripheral blood mononuclear cells and in bronchial epithelial cells [40, 41]. IL-17 also induces the secretion of IL-8, a potent neutrophil chemoattractant, in bronchial epithelial cells [42]. Taken together, these data indicate that the bronchial neutrophilia observed in our entire population of asthma patients could be due at least in part to ICS, and the loss of correlation between ICS and neutrophilia in neutrophilic patients could indicate that IL-17 drives a steroid-resistant immune mechanism in neutrophilic asthma [43].

In summary, our results contribute to a more precise characterisation of the role of airway neutrophilia in asthma, highlighting its association with lung function alterations such as increased airflow limitation, airway closure/air trapping and altered reversibility patterns. We provide preliminary evidence in support of a new endotype of asthma related to high bronchial neutrophilia, serum IgE and markers of the IL-17 pathway, and characterised clinically by increased sensitivity to perennial allergens, OCS dependence and exacerbations.

Acknowledgements: We would like to acknowledge Valentina Sorbello (Catania, Italy) for technical work, Gian Mario Massaglia (Turin, Italy) for bronchial biopsy procedures and Rosemary Allpress (Veruno, Italy) for revising the English language of the manuscript.

Support statement: This study was supported by Ricerca Sanitaria Finalizzata Regione Piemonte (2472/2007 and 38097/ 2008). Funding information for this article has been deposited with the Crossref Funder Registry.

Conflict of interest: None declared.

\section{References}

1 Panettieri RA Jr. Neutrophilic and pauci-immune phenotypes in severe asthma. Immunol Allergy Clin North Am 2016; 36: 569-579.

2 Carr TF, Zeki AA, Kraft M. Eosinophilic and non-eosinophilic asthma. Am J Respir Crit Care Med 2018; 197: 22-37.

3 Fahy JV. Eosinophilic and neutrophilic inflammation in asthma: insights from clinical studies. Proc Am Thorac Soc 2009; 6: 256-259.

4 Loza MJ, Adcock I, Auffray C, et al. Longitudinally stable, clinically defined clusters of patients with asthma independently identified in the ADEPT and U-BIOPRED asthma studies. Ann Am Thorac Soc 2016; 13: Suppl. 1, S102-S103.

5 Moore WC, Hastie AT, Li X, et al. Sputum neutrophil counts are associated with more severe asthma phenotypes using cluster analysis. J Allergy Clin Immunol 2014; 133: 1557-1563. 
6 Simpson JL, Scott R, Boyle MJ, et al. Inflammatory subtypes in asthma: assessment and identification using induced sputum. Respirology 2006; 11: 54-61.

7 Schleich FN, Manise M, Sele J, et al. Distribution of sputum cellular phenotype in a large asthma cohort: predicting factors for eosinophilic vs neutrophilic inflammation. BMC Pulm Med 2013; 13: 11.

8 Douwes J, Gibson P, Pekkanen J, et al. Non-eosinophilic asthma: importance and possible mechanisms. Thorax 2002; 57: 643-648.

9 Wenzel SE, Szefler SJ, Leung DY, et al. Bronchoscopic evaluation of severe asthma. Persistent inflammation associated with high dose glucocorticoids. Am J Respir Crit Care Med 1997; 156: 737-743.

10 Demarche S, Schleich F, Henket M, et al. Detailed analysis of sputum and systemic inflammation in asthma phenotypes: are paucigranulocytic asthmatics really non-inflammatory? BMC Pulm Med 2016; 16: 46.

11 Ricciardolo FLM, Sorbello V, Folino A, et al. Identification of IL-17F/frequent exacerbator endotype in asthma. J Allergy Clin Immunol 2017; 140: 395-406.

12 Thomson NC. Asthma and smoking-induced airway disease without spirometric COPD. Eur Respir J 2017; 49: 1602061.

13 Arron JR, Choy DF, Laviolette M, et al. Disconnect between sputum neutrophils and other measures of airway inflammation in asthma. Eur Respir J 2014; 43: 627-629.

14 Berry M, Morgan A, Shaw DE, et al. Pathological features and inhaled corticosteroid response of eosinophilic and non-eosinophilic asthma. Thorax 2007; 62: 1043-1049.

15 Woodruff PG, Khashayar R, Lazarus SC, et al. Relationship between airway inflammation, hyperresponsiveness, and obstruction in asthma. J Allergy Clin Immunol 2001; 108: 753-758.

16 Shaw DE, Berry MA, Hargadon B, et al. Association between neutrophilic airway inflammation and airflow limitation in adults with asthma. Chest 2007; 132: 1871-1875.

17 Sorkness RL, Bleecker ER, Busse WW, et al. Lung function in adults with stable but severe asthma: air trapping and incomplete reversal of obstruction with bronchodilation. J Appl Physiol 2008; 104: 394-403.

18 Quanjer PH, Ruppel GL, Langhammer A, et al. Bronchodilator response in FVC is larger and more relevant than in $\mathrm{FEV}_{1}$ in severe airflow obstruction. Chest 2017; 151: 1088-1098.

19 McCartney CT, Weis MN, Ruppel GL, et al. Residual volume and total lung capacity to assess reversibility in obstructive lung disease. Respir Care 2016; 61: 1505-1512.

20 Simpson AJ, Fowler SJ. Reclassification of bronchodilator reversibility in the U-BIOPRED adult asthma cohort using z scores. Chest 2018; 153: 1070-1072.

21 Irvin C, Zafar I, Good J, et al. Increased frequency of dual-positive $\mathrm{T}_{\mathrm{H}} 2 / \mathrm{T}_{\mathrm{H}} 17$ cells in bronchoalveolar lavage fluid characterizes a population of patients with severe asthma. J Allergy Clin Immunol 2014; 134: 1175-1186.

22 Wang $\mathrm{YH}$, Voo KS, Liu B, et al. A novel subset of $\mathrm{CD}^{+} \mathrm{T}_{\mathrm{H}} 2$ memory/effector cells that produce inflammatory IL-17 cytokine and promote the exacerbation of chronic allergic asthma. J Exp Med 2010; 207: 2479-2491.

23 Oryszczyn MP, Annesi-Maesano I, Charpin D, et al. Relationships of active and passive smoking to total IgE in adults of the Epidemiological Study of the Genetics and Environment of Asthma, Bronchial Hyperresponsiveness, and Atopy (EGEA). Am J Respir Crit Care Med 2000; 161: 1241-1246.

24 Chang Y, Al-Alwan L, Alshakfa S, et al. Upregulation of IL-17A/F from human lung tissue explants with cigarette smoke exposure: implications for COPD. Respir Res 2014; 15: 145.

25 Newcomb DC, Peebles RS Jr. Th17-mediated inflammation in asthma. Curr Opin Immunol 2013; 25: 755-760.

26 Alphonse MP, Saffar AS, Shan L, et al. Regulation of the high affinity IgE receptor (Fc epsilonRI) in human neutrophils: role of seasonal allergen exposure and Th-2 cytokines. PLoS One 2008; 3: e1921.

27 Manise M, Holtappels G, Van Crombruggen K, et al. Sputum IgE and cytokines in asthma: relationship with sputum cellular profile. PLoS One 2013; 8: e58388.

28 Redhu NS, Gounni AS. The high affinity IgE receptor (FcepsilonRI) expression and function in airway smooth muscle. Pulm Pharmacol Ther 2013; 26: 86-94.

29 Chakir J, Shannon J, Molet S, et al. Airway remodeling-associated mediators in moderate to severe asthma: effect of steroids on TGF-beta, IL-11, IL-17, and type I and type III collagen expression. J Allergy Clin Immunol 2003; 111: 1293-1298.

30 Sorbello V, Ciprandi G, Di Stefano A, et al. Nasal IL-17F is related to bronchial IL-17F/neutrophilia and exacerbations in stable atopic severe asthma. Allergy 2015; 70: 236-240.

31 Djukanovic R, Wilson JW, Britten KM, et al. Effect of an inhaled corticosteroid on airway inflammation and symptoms in asthma. Am Rev Respir Dis 1992; 145: 669-674.

32 Nguyen LT, Lim S, Oates T, et al. Increase in airway neutrophils after oral but not inhaled corticosteroid therapy in mild asthma. Respir Med 2005; 99: 200-207.

33 Brooks CR, Van Dalen CJ, Harding E, et al. Effects of treatment changes on asthma phenotype prevalence and airway neutrophil function. BMC Pulm Med 2017; 17: 169.

34 de Kluijver J, Schrumpf JA, Evertse CE, et al. Bronchial matrix and inflammation respond to inhaled steroids despite ongoing allergen exposure in asthma. Clin Exp Allergy 2005; 35: 1361-1369.

35 Foley SC, Hamid Q. Images in allergy and immunology: neutrophils in asthma. J Allergy Clin Immunol 2007; 119: $1282-1286$

36 Ward C, Pais M, Bish R, et al. Airway inflammation, basement membrane thickening and bronchial hyperresponsiveness in asthma. Thorax 2002; 57: 309-316.

37 Fukakusa M, Bergeron C, Tulic MK, et al. Oral corticosteroids decrease eosinophil and CC chemokine expression but increase neutrophil, IL-8, and IFN-gamma-inducible protein 10 expression in asthmatic airway mucosa. J Allergy Clin Immunol 2005; 115: 280-286.

38 Hauber HP, Gotfried M, Newman K, et al. Effect of HFA-flunisolide on peripheral lung inflammation in asthma. J Allergy Clin Immunol 2003; 112: 58-63.

39 Cowan DC, Cowan JO, Palmay R, et al. Effects of steroid therapy on inflammatory cell subtypes in asthma. Thorax 2010; 65: 384-390.

40 Vazquez-Tello A, Halwani R, Hamid Q, et al. Glucocorticoid receptor-beta up-regulation and steroid resistance induction by IL-17 and IL-23 cytokine stimulation in peripheral mononuclear cells. J Clin Immunol 2013; 33: 466-478.

41 Zijlstra GJ, Ten Hacken NH, Hoffmann RF, et al. Interleukin-17A induces glucocorticoid insensitivity in human bronchial epithelial cells. Eur Respir J 2012; 39: 439-445. 
42 Jones CE, Chan K. Interleukin-17 stimulates the expression of interleukin-8, growth-related oncogene-alpha, and granulocyte-colony-stimulating factor by human airway epithelial cells. Am J Respir Cell Mol Biol 2002; 26: 748-753.

43 Allen JE, Sutherland TE, Ruckerl D. IL-17 and neutrophils: unexpected players in the type 2 immune response. Curr Opin Immunol 2015; 34: 99-106. 